\title{
DETC99/DAC-8647
}

\section{WORKSPACE ANALYSIS OF 6-PRRS PARALLEL MANIPULATORS BASED ON THE VERTEX SPACE CONCEPT}

\author{
Ilian A. Bonev* and Jeha Ryu \\ Department of Mechatronics \\ Kwangju Institute of Science and Technology \\ 1 Oryong-dong, Buk-ku, Kwangju 500-712, Korea \\ Tel: +82-62-970-2389; Email: ryu@kjist.ac.kr
}

\section{ABSTRACT}

This paper presents workspace analysis of 6- $\underline{P R R S}$ parallel manipulators based on the vertex space concept. A fully geometric algorithm is described for the computation of the constant-orientation workspace, which was implemented in the $\mathrm{CAD} / \mathrm{CAM}$ system CATIA. The influence of the different design parameters on the workspace as well as on the other properties of the manipulator is discussed. Finally, examples are provided to demonstrate the usefulness of the proposed method.

\section{NOMENCLATURE}

$A_{i}$ : center of base joint $i$.

$A_{i, 0}$ : start point of rail axis $i$.

$A_{i, 1}$ : end point of rail axis $i$.

$B_{i}$ : center of platform joint $i$.

$C$ : center of the mobile reference frame (tool-tip).

$L: \quad$ stroke of the actuators.

$\mathbf{R}$ : rotation matrix defined by three Euler angles $(\phi, \theta, \psi)$.

$\ell$ : length of the legs.

$\alpha$ : maximum misalignment angle of the base joints.

$\beta$ : maximum misalignment angle of the platform joints.

\section{INTRODUCTION}

A 6-DOF fully-parallel manipulator, also called a hexapod, consists of a mobile platform connected by six legs to a base

\footnotetext{
${ }^{*}$ Currently doctoral student at Département de Génie Mécanique, Université Laval, Québec, Canada.
}

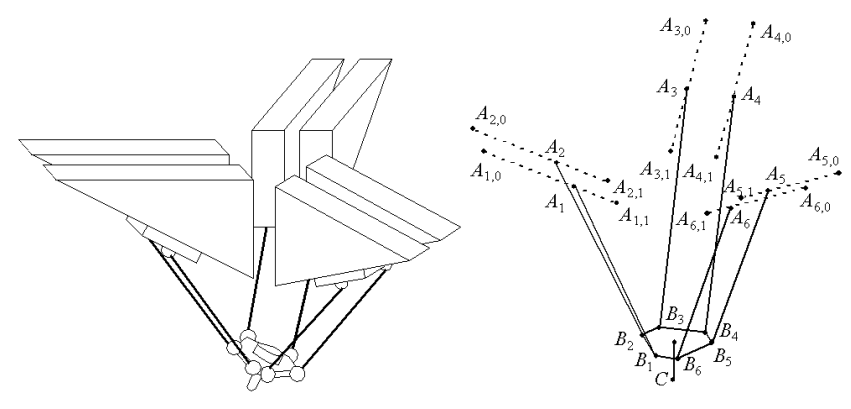

Figure 1. A typical HSM and the notation used.

through respectively spherical and universal joints. Most commonly, the base joints are fixed to the base while the legs are of variable length (e.g. Fichter, 1986; McCallion and Pham, 1979). This typical design with six RRPS serial kinematic chains, present in most existing hexapods, will be referred to as the General Parallel Manipulator (GPM).

Another common design of 6-DOF parallel manipulators uses six kinematic chains of type $\underline{P} R R S$ (Fig. 1). In this notation, $\underline{P}$ stands for an actuated prismatic joint whose axis will be called the rail axis, denoted by $A_{i, 0} A_{i, 1}, R R$ stands for a passive universal joint whose center is denoted by $A_{i}$, and $S$ stands for a passive spherical joint whose center is denoted by $B_{i}$, where $i=1, \ldots, 6$. In addition, points $O$ and $C$ are respectively the centers of the fixed base frame and of the mobile frame. For brevity, we will refer to this type of manipulator as the HexaSlide Manipulator (HSM). Examples of HSMs are the "active wrist" de-

Copyright (C) 1999 by ASME 
signed by Merlet and Gosselin (1991), the "Hexaglide" built at ETH Zurich, the "NPM" proposed by Arai et al. (1996), and the commercially available "HexaM" machine by Toyoda.

The main advantages of the HSM over the GPM come from the fact that the actuators are fixed to the base. Each leg is a small-diameter rod of constant length, attached at one end to the actuator tip and at the other to the mobile platform. Therefore, the HSM exhibits lighter moving mass and reduced risk of leg collision. The drawbacks are smaller workspace and a need for more complicated analysis due to the more complicated relationship between the mobile platform posture and the actuator lengths.

In the design of parallel manipulators, much concern is given to the workspace factor. As the complete workspace of an HSM is in a six-dimensional space for which no graphical representation exists, different types of subsets of the complete workspace are usually determined. The most commonly determined workspace is the constant-orientation workspace, which is the threedimensional volume that can be attained by point $C$ when the mobile platform is kept at a constant orientation.

A simple way of determining the workspace of a parallel manipulator is to use discretization methods (e.g. Arai et al., 1996; Conti et al., 1997; Fichter, 1986; Masory and Wang, 1992). In these methods, the workspace boundary is usually determined in a spherical coordinate system by discretizing the range of azimuth and zenith angles. For each pair of them, the ray radius is augmented until one of the constraints is violated, which is checked by solving the inverse kinematics problem. Such methods can be easily applied for any type of robot architecture and for any set of mechanical constraints. However, they are computationally intensive and give little information about the exact boundary of the workspace.

A more advanced geometric approach was first proposed by Gosselin (1990) and then again by Gosselin et al. (1992) considering only the limits of the actuators. In the first paper, horizontal cross-sections of the workspace have been determined, while in the second, the workspace edges have been defined directly. Merlet (1994) later extended this geometric approach by including the limited ranges of the joints and the risk of leg interference. Unlike the discretization methods, the geometric methods are very fast and accurate. Furthermore, they bring insight into the problem and are very useful during the design stage. All of these geometric methods were, however, concerned exclusively with the GPM. Even though, the geometric approach that was previously developed for the GPM may be applied to other architectures, compared to the GPM, the HSM presents an increased difficulty in conceiving and implementing such a geometrical algorithm due to the more complicated type of kinematic chains.

The geometric approach was first applied to an HSM by Merlet and Gosselin (1991) to compute horizontal cross-sections of the constant-orientation workspace of their "active wrist". Due to the simplifying fact, however, that all rail axes of the "active wrist" are parallel to the $z$-axis, each cross-section is obtained as the intersection of circles. A detailed study on the workspace computation of a general HSM was later performed by Allan and Gosselin (1997). Again only horizontal cross-sections have been determined, but this time obtained as the intersection of circular and elliptic arcs. In both studies, however, the authors have assumed for simplicity that the only constraint limiting the workspace of the HSMs is the actuators' stroke.

Thus, the main contribution of this article is to extend the geometric approach to the case of the HSM considering all possible constraints and directly obtaining the workspace in 3-D. In addition, a particular implementation method is suggested, which allows the integration of the whole design process in the commercially available CAD/CAM system CATIA. Based on the geometrical study of the HSM's workspace, we propose simple guidelines for its design, which illustrates the importance of the geometric approach.

The organization of this paper is as follows. In section 2, we describe the geometric method for the constant-orientation workspace of an HSM. Then, in section 3, we present in detail the general algorithm used for implementation. Based on the proposed geometrical method, we suggest in section 4 , simple design guidelines for constructing an HSM with desired workspace characteristics. In section 5, we present examples of the constant-orientation workspace of an HSM and its equivalent GPM. Further suggestions for extending the proposed approach for the computation of other types of workspaces are given in section 6 , and the final section 7 presents the conclusions.

\section{GEOMETRIC STUDY OF THE WORKSPACE}

In order to describe a geometric method for computing the workspace of an HSM, it is necessary to establish geometric models for all the constraints that determine the workspace. The basic idea is first to regard all kinematic chains as independent and then to consider their interdependence (Gosselin, 1990).

Thus, for a constant orientation of the mobile platform, let us define the vertex space $i$ (the term is shared from $\mathrm{Ji}, 1994$ ) as the volume that can be attained by vertex $B_{i}$ from chain $i$, ignoring the constraints imposed by all other kinematic chains. The constraints that determine each vertex space are (i) the leg's length, (ii) the serial-chain singularity, (iii) the slider-leg interference, (iv) the ranges of the base and mobile platform joints, and finally (v) the actuator's stroke. Next, we will investigate all the constraints to finally construct the vertex space, assuming that all serial chains are identical.

\subsection{Leg's Length}

Let the length of a leg be $\ell$. Thus, when point $A_{i}$ coincides with point $A_{i, 0}$, the set of points reachable by $B_{i}$ is a sphere $S_{i}$ of radius $\ell$ and center $A_{i, 0}$ (Fig. 2). 


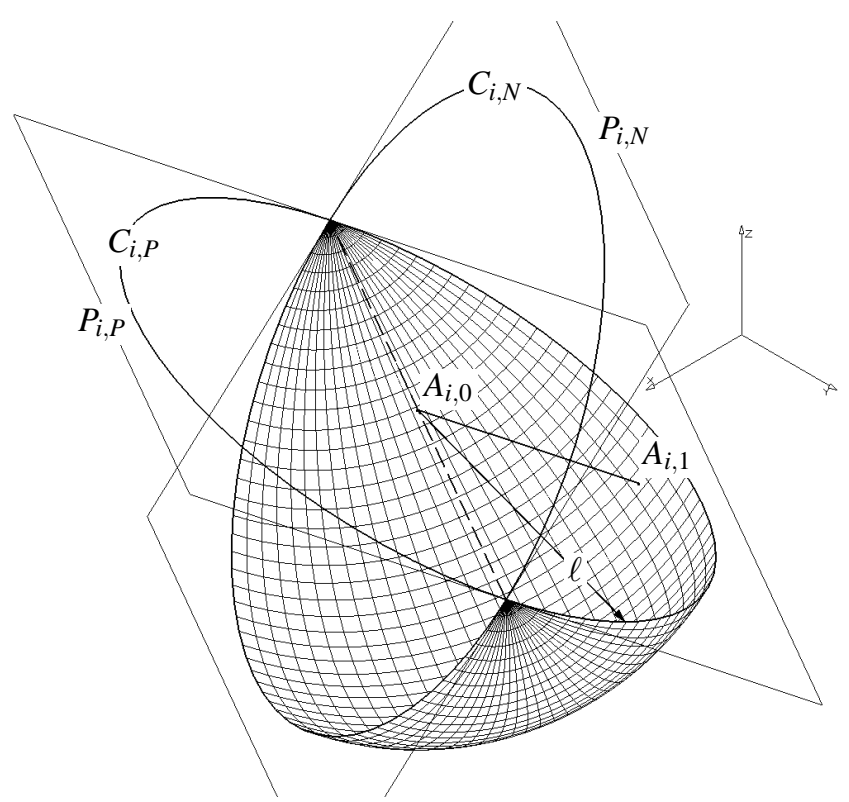

Figure 2. The lune modeling constraints 2.1 through 2.3.

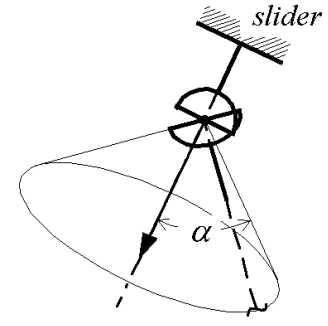

(a)

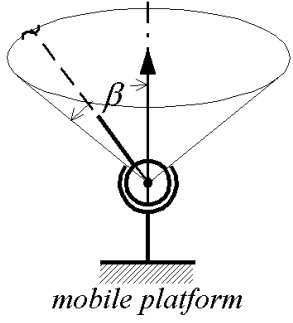

(b)
Figure 3. Ranges of motion of the (a) base and (b) platform joints.

\subsection{Serial-Chain Singularity}

A particular characteristic of HSMs is that their serial chains have a singularity at configurations where a leg is perpendicular to its corresponding rail axis. In this singularity, the two branches of the inverse kinematics of a serial chain meet and the mobile platform loses one degree of freedom (Gosselin and Angeles, 1990). Since passing through such a singularity is undesirable, the motion of each leg should be restricted so that the angle between vectors $\mathbf{A}_{i} \mathbf{B}_{i}$ and $\mathbf{A}_{i, 0} \mathbf{A}_{i, 1}$ be always in only one of the two ranges $\left[0^{\circ}, 180^{\circ}\right)$ or $\left(90^{\circ}, 180^{\circ}\right]$. Hence, we split the sphere $S_{i}$ by a plane $P_{i, N}$, normal to the rail axis and passing through point $A_{i, 0}$. Without loss of generality, we take the hemisphere from the side of the rail axis (Fig. 2). The great circle formed by the intersection of $S_{i}$ with that plane will be denoted by $C_{i, N}$.

This constraint has a very peculiar role, and if it is not considered, the workspace analysis changes significantly. At the end of this section, we will discuss what will occur if the constraint

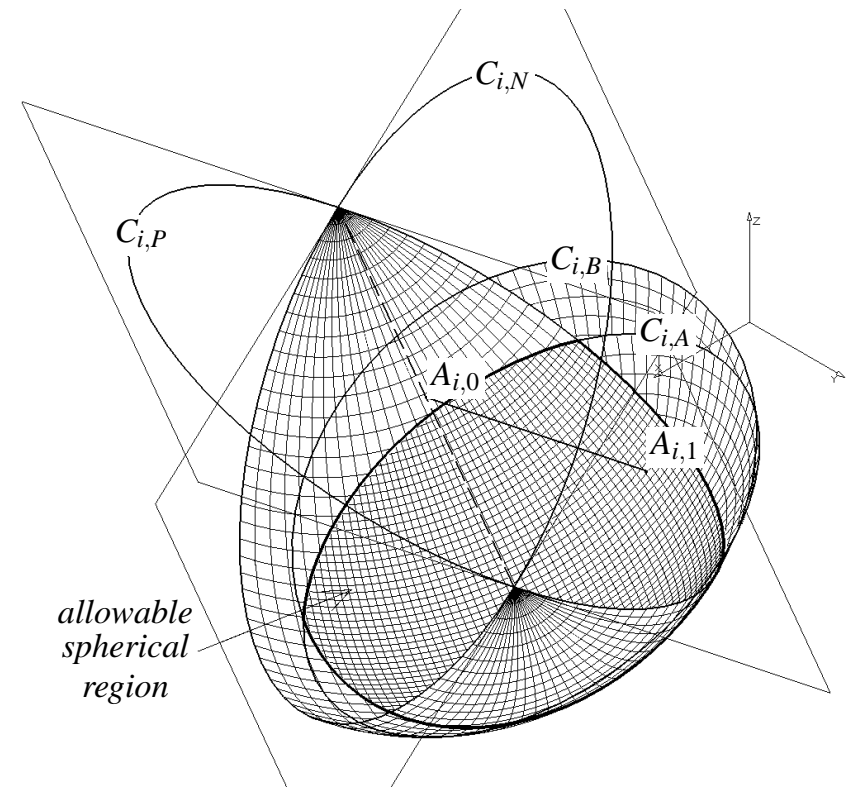

Figure 4. The lune and the two spherical caps.

is not considered. At this point, let us just note that all existing HSMs are designed to exclude singularities in their serial chains.

\subsection{Slider-Leg Interference}

We assume that the prismatic joints are comprised of linear motion (LM) guides, which is the case in most existing HSMs. If the actuators are of telescopic type (as in the "active wrist"), then this constraint does not exist and the workspace analysis is simpler. In addition, the workspace is much larger, but at the expense of a lower overall stiffness of the manipulator.

The sliders that move along the LM guides are relatively wide with a planar face. To avoid a slider-leg collision, the leg should be only in one of the half-spaces separated by the plane $P_{i, P}$, parallel to the slider face and passing through point $A_{i, 0}$. Let $\mathbf{N}_{i}$ be the unit vector normal to that plane and in the direction of the allowable half-space. The great circle formed by the intersection of the plane with $S_{i}$ will be denoted by $C_{i, P}$. Thus, the hemisphere is redefined to a $90^{\circ}$ lune (Fig. 2).

\subsection{Range of the Base Joint}

The physical constraints that limit the range of a passive joint can be modeled by a general conical surface whose vertex is the center of the joint. We already mentioned that the legs are attached to the sliders through universal joints but in practice spherical joints are often used instead. Thus, we chose to model the constraint imposed by the base joint as a circular cone (Fig. 3a). If indeed, U-joints are used, then a better model would be a pyramid as used in (Merlet, 1994), which, however, will in- 


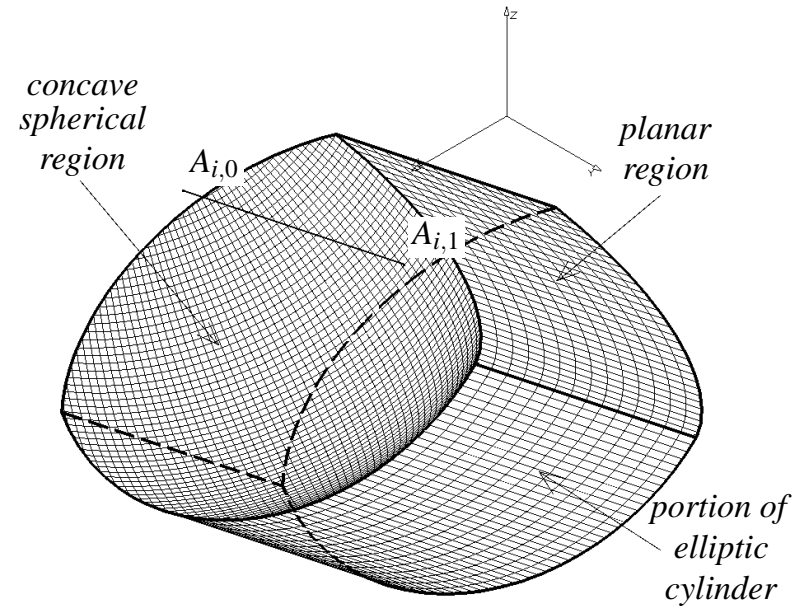

Figure 5. Vertex space i.

evitably make the workspace analysis slightly more complicated.

Let $\alpha$ be the maximum misalignment angle of the base joints $\left(\alpha<90^{\circ}\right)$ and let $\mathbf{j}_{A_{i}}$ be the unit vector along the axis of symmetry of the joint at point $A_{i}$. Then, the allowable region for point $B_{i}$ consists of a spherical cap of radius $\ell$ and center $A_{i}$. This cap will be denoted by $K_{i, A}$, and its base circle by $C_{i, A}$ (Fig. 4).

\subsection{Range of the Mobile Platform Joint}

The same cone model will be used for the platform spherical joints. Let $\beta$ be the maximum misalignment angle of the platform joints $\left(\beta<90^{\circ}\right)$ and let $\mathbf{j}_{B_{i}}^{\prime}$ be the unit vector along the axis of symmetry of the joint with center $B_{i}$ with respect to the mobile frame (Fig. 3b). Then, the allowable region for point $A_{i}$, referred to the mobile frame, consists of a spherical cap of radius $\ell$ and center $B_{i}$. Let $\mathbf{j}_{B_{i}}$ be the opposite unit vector, and with respect to the base frame, defined as:

$$
\mathbf{j}_{B_{i}}=-\mathbf{R j}_{B_{i}}^{\prime}
$$

Thus, with respect to the base frame, point $B_{i}$ is located on an identical spherical cap of radius $\ell$ but center $A_{i}$ (Fig. 4). This cap will be denoted by $K_{i, B}$, and its base circle by $C_{i, B}$.

\subsection{Actuator's Stroke}

The allowable spherical region for point $B_{i}$, when point $A_{i}$ is fixed, is the intersection of the lune and the two spherical caps defined previously (the square-hatched region in Fig. 4). Now, since point $A_{i}$ can move between points $A_{i, 0}$ and $A_{i, 1}$, the allowable spatial region for vertex $B_{i}$ is the volume swept by the allowable spherical region along the directrix $\mathbf{A}_{i, 0} \mathbf{A}_{i, 1}$. This volume is vertex space $i$ (Fig. 5). Let us also define the vertex space obtained for an unlimited range of the platform joint as the $\max$ - imum vertex space. Note that the latter is independent from the orientation of the mobile platform.

After the six vertex spaces have been defined, we must consider the fact that all points $B_{i}$ are rigidly fixed to the mobile platform. Let us call this the closure constraint. Since the mobile platform is kept at a constant orientation, the allowable spatial region for point $C$ - taking into account the restrictions imposed by only kinematic chain $i$-is obtained by translating vertex space $i$ along the vector $\mathbf{B}_{i} \mathbf{C}$. Thus, the intersection of all six translated vertex spaces is the workspace of the HSM.

Now, for more precise workspace determination, the next constraints to be considered should be the leg interference and the singularity configurations. Both of these constraints relate to the geometric relationships among the six line segments $A_{i} B_{i}$. For the GPM case, Merlet (1994) has shown that the leg interference can be generally modeled by quadratic surfaces, which divide the workspace into separate volumes. Unfortunately, the expressions defining the six line segments $A_{i} B_{i}$ of an HSM as functions of the platform orientation are too complicated to involve any algebraic study of these two constraints. Thus, the only way to consider them is by using a numerical method following the application of the proposed geometric algorithm.

Finally, let us see what happens if we do not consider the serial-chain singularity constraint. Instead of a $90^{\circ}$ lune, we will have a hemisphere, and the allowable spherical regions (recall Fig. 4) may have portions from both sides of plane $P_{i, N}$. Sweeping such a spherical surface can only be implemented by sweeping separately each of its two portions and then taking the union of the two swept volumes. Consequently, it may happen that the workspace of the HSM consists of two separate volumes.

\section{IMPLEMENTATION ALGORITHMS}

As we saw, the constant-orientation workspace of an HSM is the intersection of the six translated vertex spaces. Hence, the following two observations can be made.

Observation 1. The boundary of the constant-orientation workspace consists of portions of spheres, right circular cylinders, elliptic cylinders, and planes.

Observation 2. The edges of the constant-orientation workspace consist of line segments, circular and elliptic arcs, and segments of spatial curves of order 4.

Next, we will propose an algorithm for determining each vertex space. The idea is to obtain explicitly the contour of the allowable spherical region and then to construct the boundary representation of the vertex space. We assume that $\beta \geq \alpha$.

\section{Algorithm for the Vertex Space:}

S1. If circles $C_{i, A}$ and $C_{i, B}$ coincide (i.e. $\alpha=\beta$ and $\mathbf{j}_{A_{i}}=\mathbf{j}_{B_{i}}$ ) then do not consider $C_{i, B}$ further. 


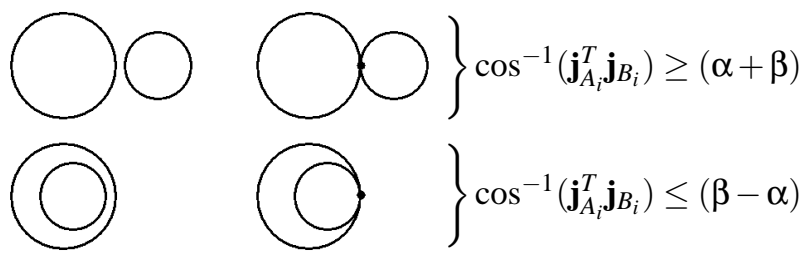

Figure 6. The possible cases where the number of intersection points between $C_{i, A}$ and $C_{i, B}$ is less than two.

S2. Calculate the intersection points between all pairs of circles $C_{i, N}, C_{i, P}, C_{i, A}$, and $C_{i, B}$, storing each point into lists corresponding to each circle.

S3. For each circle, order the list of intersection points. If two or three points coincide (i.e. three or all of the circles have a common point) then erase all but one.

S4. For each circle, if its list has more than one intersection point, calculate the center point of each arc connecting two successive intersection points. If this center point lies on the lune and on the two spherical caps then construct the arc and put it into the list defining the contour.

S5. For circles $C_{i, A}$ and $C_{i, B}$, if their lists have one or no intersection points (see Fig. 6), then check whether $\cos ^{-1}\left(\mathbf{j}_{A_{i}}^{T} \mathbf{j}_{B_{i}}\right) \leq(\beta-\alpha)$. If this is not true, then stop (i.e. vertex space $i$ and, consequently, the workspace do not exist for this orientation). Otherwise, put $C_{i, A}$ in the list and go to step 7 (i.e. the contour is simply circle $C_{i, A}$ ).

S6. Order the list of arcs that define the contour.

S7. Define the spherical patch bounded by the contour (i.e. the allowable spherical region).

S8. Translate a duplicate of that patch along $\mathbf{A}_{i, 0} \mathbf{A}_{i, 1}$.

S9. Create the side boundary of the vertex space by offsetting the contour along $\mathbf{A}_{i, 0} \mathbf{A}_{i, 1}$.

S10. Create a volume from the three boundaries.

Depending on the available geometric tools and on the desired visualization technique, the sequence of steps for constructing the workspace can be different. If no geometric libraries are available, then the horizontal cross-sections of the workspace can be determined as in (Merlet, 1994). Alternatively, the edges of the workspace can be calculated directly as in (Gosselin et al., 1992) to obtain a simplified wireframe model. Finally, if a geometric library capable of performing Boolean operations on solids is available, then the workspace can be directly determined in 3-D as a solid model.

We implemented our approach in the CAD/CAM system CATIA using its IUA application programming interface. This choice was motivated by (i) CATIA's advanced visualization capabilities and (ii) the possibility for integrating the entire design process in one CAD/CAM system.

\section{DESIGN CONSIDERATIONS}

As the constant-orientation workspace is the intersection of the six vertex spaces, it is easy to make the following observation.

Observation 3. The constant-orientation workspace cannot be larger than the maximum vertex space.

Based on the geometrical description of the HSM's workspace, we will now study the design parameters that influence it. To allow a systematic study, we will classify the parameters into three groups. The first group consists of the actuators' stroke $(L)$, the legs' length $(\ell)$, and the ranges of the joints $(\alpha, \beta)$. The second group includes the arrangement of the LM guides $\left(\mathbf{O A}_{i, 0}\right.$, $\left.\mathbf{O A}_{i, 1}\right)$ and the platform joints $\left(\mathbf{C B}_{i}^{\prime}\right)$. The third group consists of the orientation of the joints $\left(\mathbf{j}_{A_{i}}, \mathbf{j}_{B_{i}}^{\prime}\right)$. Finally, we will chose the performance criteria as (i) the volume and (ii) the shape of the workspace at a given orientation, and (iii) their variation as a function of the orientation.

\subsection{First Group of Design Parameters}

The first group of design parameters relates to the volume and shape of the vertex spaces. The main components of an HSM are the linear actuators or-in the workspace contexttheir stroke $L$. Naturally, the larger the stroke, the larger the workspace.

The next components are the legs. In general, the longer the legs, the bigger the workspace volume. When $\ell \gg L$, the volume of the workspace decreases rapidly at greater orientations. When $\ell<L$, the workspace shape becomes slightly simpler. Shorter legs also prevent error amplification.

The last components are the joints or-in particular-their ranges. In order to maximize the vertex space, the spherical cap $K_{i, A}$ should cover completely the lune. For an angle of $\alpha=90^{\circ}$, the spherical cap may cover completely the lune, while for an angle of $\alpha \leq 50^{\circ}$, the spherical cap may cover no more than two thirds of it.

As the platform orientation changes, the spherical cap $K_{i, B}$ covers different parts of the constant intersection of the lune and the cap $K_{i, A}$. The vertex space will be maximal when $K_{i, B}$ covers completely that intersection. The larger the value of $\beta$, the higher the range of orientations at which all vertex spaces exist.

\subsection{Second Group of Design Parameters}

The arrangement of the LM guides defines the orientation and the initial position of the vertex spaces. This is the most distinguishing characteristic of an HSM, which determines the 


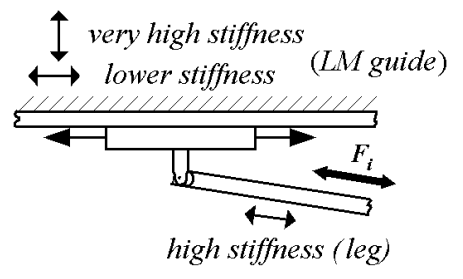

(a)

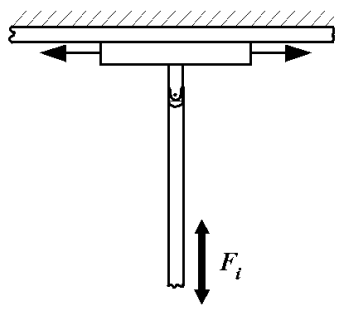

(b)
Figure 7. Two extreme configurations of a serial chain characterized by (a) lower and (b) higher stiffness and resolution at the end point $B_{i}$.

shape (and volume) of its workspace. For example, if the rail axes are mutually parallel and arranged along a cylindrical surface (as in the "active wrist"), then the workspace is close to a prism. If the rail axes are mutually parallel but coplanar (as in the "Hexaglide"), then the workspace is close to a semi-cylinder topped with two quarter-spheres.

The arrangement of the LM guides also affects the resolution of the HSM. Note that when vertex $B_{i}$ is close to the cylindrical boundary of the vertex space, i.e. when serial chain $i$ is nearly at singular configuration, large actuator displacements lead to small platform motions (Carretero et al., 1997). On the contrary, when vertex $B_{i}$ is close to the center of the planar boundary of the vertex space, i.e. when the leg is almost collinear with the rail axis, the actuator displacements lead to compatible platform motions. Thus, if high resolution is aimed, the rail axes should be arranged in such a way that the resulting boundaries of the workspace be mainly cylindrical (e.g. as in the "Hexaglide"). Furthermore, the stiffness of such an HSM will be higher since it will be less affected by the axial compliance of the LM guides (Fig. 7).

The reasons for the variation of the workspace at different orientations are (i) the variation of the vertex spaces due to the variation of the platform joints' orientations, and (ii) the variation of the vectors $\mathbf{B}_{i} \mathbf{C}$. The variation of the workspace is also dependent on the initial arrangement of the LM guides. For the first reason, we already mentioned that increasing the range of the platform joints is the main way to reduce the variation of the vertex spaces. As for the second reason, recall that the arrangement of the platform joints defines the vectors $\mathbf{B}_{i} \mathbf{C}$ along which the vertex spaces are finally translated. Hence, naturally, the smaller the length of these vectors, the smaller the variation of the workspace. Decreasing the length of these vectors, however, increases the risk of leg interference and reduces the rotational resolution of the mobile platform.

\subsection{Third Group of Design Parameters}

The final parameters to be considered define the installation of the joints. Masory and Wang (1992) have already suggested how to install the joints of a GPM in order to obtain a larger workspace. A similar guideline is to be followed for the HSM.

For the base joints, each vector $\mathbf{j}_{A_{i}}$ is to be selected so that it lies in the plane passing through the rail axis and perpendicular to $P_{i, P}$. In general, the acute angle between a leg and its rail axis is maximum when the mobile platform is in its lowest position and minimum when the platform is in its highest position. Hence, the angle between $\mathbf{j}_{A_{i}}$ and the rail axis should be the average of these maximum and minimum angles. As for the platform joints, vector $\mathbf{j}_{B_{i}}^{\prime}$ should be selected in such a way that $\mathbf{j}_{A_{i}}=\mathbf{j}_{B_{i}}$ at the reference orientation, since usually the workspace at this orientation should be maximum.

\section{EXAMPLES}

To illustrate our geometric method, we take as an example a "HexaM" type of HSM (Fig. 1) whose data are given in the Appendix.

In our implementation, we adopt the choice of modified Euler angles introduced in (Bonev and Ryu, 1999) to represent the orientation of the mobile platform. For this choice, we rotate first the mobile platform around the base $z$-axis by an angle $-\phi$, then around the base $y$-axis by an angle $\theta$, then around the base $z$-axis by an angle $\phi$ and finally around the mobile $z^{\prime}$-axis by an angle $\psi$. Defined in this way, angle $\psi$ is the roll angle, angle $\theta$ is the tilt angle, and angle $\phi$ is the angle between the base $x$-axis and the projection of the mobile $z^{\prime}$-axis onto the base $x y$-plane. The resultant $3 \times 3$ rotation matrix $\mathbf{R}$ is defined as:

$$
\mathbf{R}=\mathbf{R}_{z}(\phi) \mathbf{R}_{y}(\theta) \mathbf{R}_{z}(\psi-\phi)
$$

where $\mathbf{R}_{z}(\cdot)$ and $\mathbf{R}_{y}(\cdot)$ are basic rotation matrices. These angles have the property that the constant-orientation workspace for a fixed direction of the mobile $z^{\prime}$-axis $(\phi, \theta)$ is largest at $\psi=0^{\circ}$.

We present here two examples of the HSM's workspace. The first one is at the reference orientation (Fig. 8), $\phi=\theta=\psi=0^{\circ}$, and the second is at a relatively extreme orientation (Fig. 9), $\phi=$ $135^{\circ}, \theta=30^{\circ}, \psi=0^{\circ}$. It is interesting to note that the volume of the workspace at the reference orientation $\left(0.328 \mathrm{~m}^{3}\right)$ is almost equal to the cube of the actuators' stroke $\left(L^{3}=0.343 \mathrm{~m}^{3}\right)$ and less than one half of the volume of the maximum vertex space $\left(0.720 \mathrm{~m}^{3}\right)$. Note also the complex shape of the workspace due to the fact that $\ell>L$. Also, at this orientation, the workspace is not affected by the joint ranges. As for the second example, the workspace is severely limited by the range of one of the platform joints, which accounts for the portions of elliptic cylinder in the workspace boundary.

Finally, to illustrate the known drawbacks of HSMs for having a smaller workspace than GPMs, we present an example of the workspace of a GPM, equivalent to the HSM used in the previous examples. In that GPM, the centers of the base joints are chosen as points $A_{i, 0}$ of the HSM, and the range of the actuators is $[\ell, \ell+L]$. All other parameters are the same. 


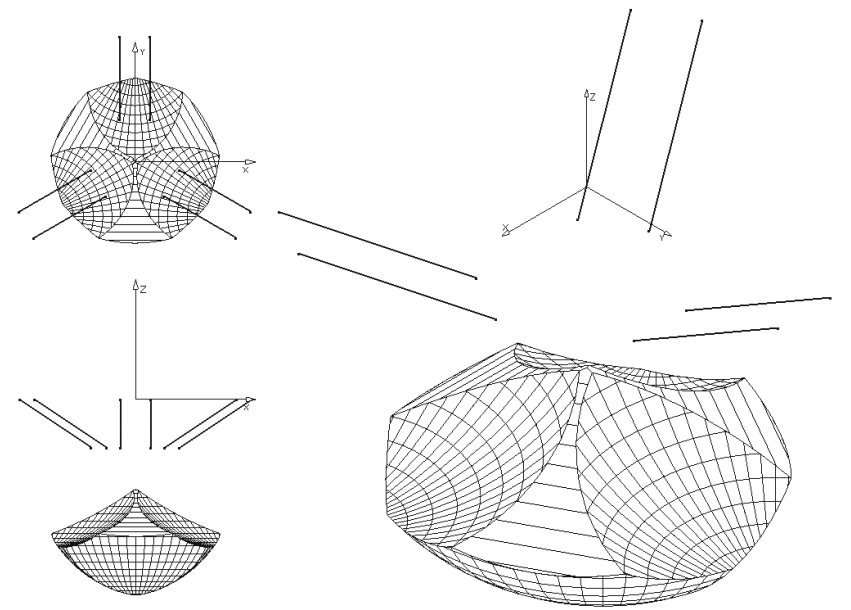

Figure 8. The workspace of the HSM for the reference orientation. The volume of the workspace is $0.328 \mathrm{~m}^{3}$.

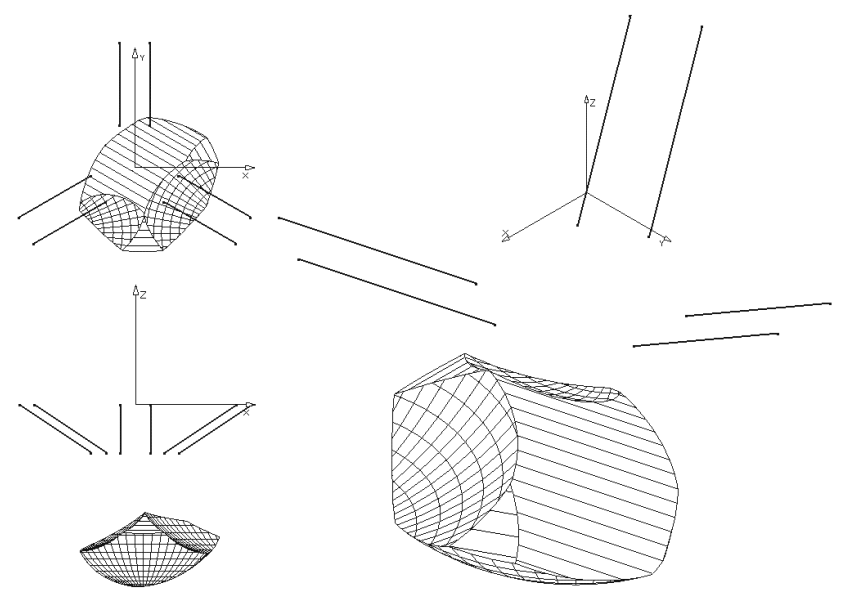

Figure 9. The workspace of the HSM for an extreme orientation. The volume of the workspace is $0.154 \mathrm{~m}^{3}$.

Indeed, the volume of the workspace of the equivalent GPM for the reference orientation is $0.447 \mathrm{~m}^{3}$, or with a third bigger than that of the HSM. A substantial portion of that increase is, however, situated in the sharp-edged top regions of the workspace (see Fig. 10), which are hardly expected to be utilized in practice.

As already mentioned, a program was written in CATIA V4R1.9 using its IUA application programming interface for computing the constant-orientation workspace of an HSM. A similar-yet much simpler-program was also written for the GPM case. The programs were run on a $175 \mathrm{MHz}$ SGI Indigo 2 workstation with $256 \mathrm{Mb}$ RAM. The computation time for the HSM case was established at about 2 min, while for the GPM case, it was only $1 \mathrm{~min}$. Note that since the maximum vertex
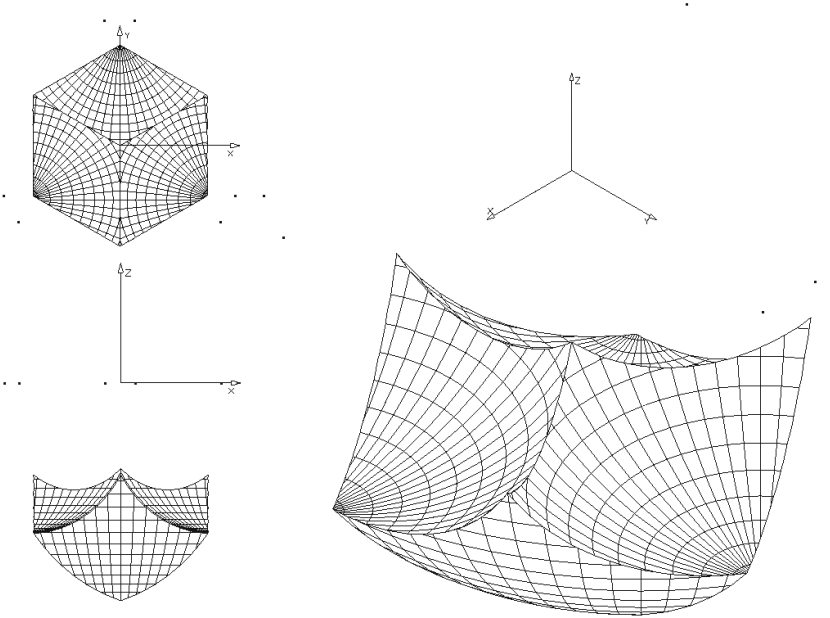

Figure 10. The workspace of the equivalent GPM for the reference orientation. The volume of the workspace is $0.447 \mathrm{~m}^{3}$.

spaces are constant, the computation time can be reduced by prestoring them.

\section{FURTHER WORK AND SUGGESTIONS}

Based on the vertex space approach, we may suggest the foundations of other geometrical methods for determining the different types of workspaces. Of great practical interest is the total orientation workspace defined as the spatial region in which the mobile platform can reach any orientation within a given range (Merlet et al., 1998). Particularly, the user may be interested in the workspace for the range of orientations defined by $\phi \in\left[0^{\circ},+360^{\circ}\right), \theta \in\left[0^{\circ}, \theta_{\max }\right]$, and $\psi=0^{\circ}$. For this range, each vertex $B_{i}$ describes an identical spherical region with center $C$. Similarly, it is possible to find an approximated reduced spherical cap $K_{i, B}$, which models the platform joint range for the total range of orientations. A given point belongs to the total orientation workspace if each spherical region is fully included in the corresponding reduced vertex space.

For some particular HSM designs there is more than one slider moving along the same guideway (e.g. for the "Hexaglide"). Therefore, a geometric model should be developed for the potential slider-slider interference. In the case of the "Hexaglide", for each pair of legs, this model will be simply a tabulated cylindrical surface, which may exclude a portion of the constant-orientation workspace.

Finally, in terms of design, we saw that the arrangement of the rail axes is the main factor influencing the shape of the workspace and the resolution of the machine. Therefore, it may be useful to create a reconfigurable HSM in which one or more parameters control the arrangement of the rail axes. 


\section{CONCLUSIONS}

A geometric algorithm for the determination of the constantorientation workspace of 6-PRRS parallel robots was introduced. The algorithm was implemented in the CAD/CAM system CATIA using its application programming interface. Design criteria were proposed based on the geometric model of the workspace. Finally, an example was presented for the workspace of a "HexaM" type of manipulator.

It became clear that the frequently mentioned drawback of HSMs for having a smaller workspace than GPMs is compensated by an increased resolution. Combined with the main advantages of HSMs for having an improved dynamics, the HSM emerges as indispensable for applications where accuracy dominates over workspace requirements.

\section{REFERENCES}

Allan, J.-F., and Gosselin, C. M., 1997, "Analyse cinématique d'un manipulateur parallèle spatial à 6 degrés de liberté," Research Report, December, Université Laval, Canada.

Arai, T., Tanikawa, T., Merlet, J.-P., and Sendai, T., 1996, "Development of a New Parallel Manipulator With Fixed Linear Actuator," ASME Japan/USA Symposium on Flexible Automation, Boston, Massachusetts, Vol. 1, pp. 145-149.

Bonev, I. A., and Ryu, J., 1999, "Orientation Workspace Analysis of 6-DOF Parallel Manipulators," Proceedings of the ASME Design Engineering Technical Conferences, Las Vegas, Nevada, DETC99/DAC-8646.

Carretero, J. A., Nahon, M., Buckham, B., and Gosselin, C. M., 1997, "Kinematic Analysis of a Three-DOF Parallel Mechanism for Telescope Applications," Proceedings of the ASME Design Engineering Technical Conferences, Sacramento, California, DETC97/DAC-3981.

Conti, J. P., Clinton, C. M., Zhang, G., and Wavering, A. J., 1997, "Dynamic Variation of the Workspace of an Octahedral Hexapod Machine During Machining," Technical Research Report, TR 97-28, ISR, University of Maryland, Maryland.

Fichter, E. F., 1986, "A Stewart Platform-Based Manipulator: General Theory and Practical Construction," The International Journal of Robotics Research, Vol. 5, No. 2, pp. 157-182.

Gosselin, C. M., 1990, "Determination of the Workspace of 6-DOF Parallel Manipulators," ASME Journal of Mechanical Design, Vol. 112, No. 2, pp. 157-182.

Gosselin, C. M., and Angeles, J., 1990, "Singularity Analysis of Closed-Loop Kinematic Manipulators," IEEE Transactions on Robotics and Automation, Vol. 6, No. 3, June, pp. 281-290.

Gosselin, C. M., Lavoie, E., and Toutant, P., 1992, “An Efficient Algorithm for the Graphical Representation of the ThreeDimensional Workspace of Parallel Manipulators," Proceedings of the ASME 22nd Biennial Mechanisms Conference, Scottsdale, Arizona, Vol. 45, pp. 323-328.

Ji, Z., 1994, "Workspace Analysis of Stewart Platforms via
Vertex Space," Journal of Robotic Systems, Vol. 11, No. 7, pp. 632-639.

Masory, O., and Wang, J., 1992, "Workspace Evaluation of Stewart Platforms," Proceedings of the ASME 22nd Biennial Mechanisms Conference, Scottsdale, Arizona, Vol. 45, pp. $337-$ 346.

McCallion, H., and Pham, D. T., 1979, “The Analysis of a Six Degree of Freedom Work Station For Mechanized Assembly," Proceeding of the 5th World Congress on Theory of Machines and Mechanisms, Montreal, Canada, pp. 611-616.

Merlet, J.-P., 1994, “Détermination de l'espace de travail d'un robot parallèle pour une orientation constante," Mechanism and Machine Theory, Vol. 29, No. 8, pp. 1099-1113.

Merlet, J.-P., and Gosselin, C. M., 1991, "Nouvelle architecture pour un manipulateur parallèle à 6 degrés de liberté," Mechanism and Machine Theory, Vol. 2, No. 26, pp. 77-90.

Merlet, J.-P., Gosselin, C. M., and Mouly, N., 1998, "Workspaces of Planar Parallel Manipulators," Mechanism and Machine Theory, Vol. 33, No. 1/2, pp. 7-20.

\section{APPENDIX: DATA FOR THE HSM}

Table 1 shows the data for the "HexaM" type of HSM used in the examples given in section 5. In addition, $\ell=900 \mathrm{~mm}$, $L=700 \mathrm{~mm}, \alpha=\beta=50^{\circ}$.

Table 1. Geometry of the HSM (all units are in [mm]).

\begin{tabular}{|c|c|c|c|c|c|}
\hline$i$ & $\mathbf{O A}_{i, 0}$ & $\mathbf{O A}_{i, 1}$ & $\mathbf{C B}_{i}^{\prime}$ & $\mathbf{j}_{A_{i}}=-\mathbf{j}_{B_{i}}^{\prime}$ & $\mathbf{N}_{i}$ \\
\hline \multirow{3}{*}{1} & $\overline{[-738.035]}$ & $\overline{\Gamma-213.035}$ & $-51.507]$ & {$[0.433]$} & $\overline{[-0.433]}$ \\
\hline & -553.122 & -250.013 & -156.755 & 0.250 & -0.250 \\
\hline & 0.000 & {$[-350.000$} & 200.000 & -0.866 & {$[-0.866]$} \\
\hline \multirow{3}{*}{2} & {$[-848.035]$} & {$[-323.035$} & {$[-161.507]$} & {$[0.433]$} & {$[-0.433]$} \\
\hline & -362.596 & -59.487 & -33.771 & 0.250 & -0.250 \\
\hline & 0.000 & {$[-350.000$} & 200.000 & -0.866 & {$[-0.866]$} \\
\hline \multirow{3}{*}{3} & {$[-110.000]$} & {$[-110.000$} & {$[-110.000]$} & 0.000 & 0.000 \\
\hline & -915.718 & -309.500 & -122.984 & 0.500 & -0.500 \\
\hline & 0.000 & -350.000 & 200.000 & -0.866 & -0.866 \\
\hline \multirow{3}{*}{4} & 110.000 & $110.000^{-}$ & 110.0007 & $0.000^{-}$ & $0.000]$ \\
\hline & -915.718 & -309.500 & -122.984 & 0.500 & -0.500 \\
\hline & 0.000 & -350.000 & 200.000 & -0.866 & -0.866 \\
\hline \multirow{3}{*}{5} & 848.035 & 323.035 & 161.507 & $-0.433]$ & 0.433 \\
\hline & -362.596 & -59.487 & -33.771 & 0.250 & -0.250 \\
\hline & 0.000 & {$[-350.000$} & 200.000 & -0.866 & {$[-0.866]$} \\
\hline \multirow{3}{*}{6} & {$[738.035]$} & 213.035 & 51.507 & {$[-0.433]$} & 0.433 \\
\hline & -553.122 & -250.013 & -156.755 & 0.250 & -0.250 \\
\hline & 0.000 & -350.000 & 200.000 & -0.866 & -0.866 \\
\hline
\end{tabular}

